\title{
Neoadjuvant Chemotherapy with Capecitabine and Temozolomide for Unresectable Pancreatic Neuroendocrine Tumor
}

\author{
Sumana Devata Edward J. Kim \\ Department of Internal Medicine, University of Michigan Medical Center, \\ Ann Arbor, Mich., USA
}

\section{Key Words}

Pancreatic neuroendocrine tumor · Temozolomide $\cdot$ Capecitabine

\begin{abstract}
Pancreatic neuroendocrine tumors (PNETs) are relatively rare tumors that arise in the endocrine cells of the pancreas. Historically, somatostatin analogues have been used in this disease primarily for symptom control and, to a limited extent, disease stability. More recently, sunitinib and everolimus have been approved for advanced stage PNETs based on a survival benefit. However, both agents have $a<10 \%$ actual response rate and cause nontrivial side effect profiles that limit duration of therapy. In locally advanced disease, there is a paucity of data to support an optimal neoadjuvant approach with the expectation of down-staging to allow for curative resection. We describe in this case a young woman who was successfully down-staged using a chemotherapy regimen of capecitabine and temozolomide with minimal toxicity.
\end{abstract}

\section{Case}

A 31-year-old woman presented with a 6-month history of diarrhea, hyperglycemia requiring insulin, weight loss and a 2-month history of icterus and jaundice. Laboratory evaluation revealed elevated liver function tests including alanine aminotransferase (ALT) of $456 \mathrm{IU} / \mathrm{l}$, aspartate aminotransferase (AST) of $233 \mathrm{IU} / \mathrm{l}$, alkaline phosphatase of $791 \mathrm{IU} / \mathrm{l}$, and total bilirubin of $4.4 \mathrm{mg} / \mathrm{dl}$. Abdominal ultrasound showed a pancreatic head mass measuring $5 \times 5 \times 5.5 \mathrm{~cm}$ concerning for malignancy. Computed tomography (CT) of the abdomen confirmed a large pancreatic head mass measuring $5.7 \times 5.7 \times 6.5 \mathrm{~cm}$ obstructing both the pancreatic and common bile ducts. The mass also caused impingement of the superior mesenteric vein (SMV) and contacted the superior mesenteric artery (SMA). No liver lesions were identified, although several portocaval lymph nodes were noted to 
be borderline enlarged. Initial attempt to obtain a tissue diagnosis was performed at the time of endoscopic placement of a biliary stent and brushings revealed only reactive ductal epithelium, which was negative for malignancy. She subsequently underwent an endoscopic ultrasound-guided fine needle aspiration which confirmed a pancreatic neuroendocrine tumor (PNET) with neoplastic cells positive for synaptophysin and chromogranin. Serum chromogranin A level was noted to be elevated at $438 \mathrm{mg} / \mathrm{ml}(\mathrm{nl}<225 \mathrm{ng} / \mathrm{ml})$.

Given the absence of evidence for distant disease, the patient was offered surgery and underwent an exploratory laparotomy. Intraoperative visualization of the tumor revealed more extensive vessel involvement than appreciated by CT imaging. There was encasement of the SMV with tumor growth around numerous branches of the SMA and extension to the junction of the right renal vein and IVC. Based on this appearance, an attempt at resection was aborted as a resection with SMV reconstruction was not felt to be possible. Her postoperative course was complicated by a pulmonary embolism, which was attributed to recent surgery and underlying malignancy. Medical oncology was consulted for evaluation and recommendations for systemic therapy. Standard options including octreotide, sunitinib, and everolimus were considered and offered to the patient. However, given the patient's age and disease limited to primary site, it was agreed that the goal of therapy, in this case, should be to achieve tumor shrinkage sufficient to allow a repeat attempt at curative surgical resection. The patient was offered and accepted chemotherapy with a regimen of capecitabine and temozolomide. She ultimately received 8 cycles which she tolerated well while maintaining an excellent performance status. Toxicity was limited to one episode of emesis with each of the first two cycles. Interval imaging done every 2 cycles showed continuous response to therapy with the tumor ultimately measuring 1.9 $\times 3.8 \mathrm{~cm}$ after cycle $8(\underline{\mathrm{fig}} \mathbf{1})$. Although there appeared to be persistent abutment with the SMV, there was resolution of impingement of the vein and importantly a clear fat plane was seen around the SMA.

Based on this significant response to chemotherapy, the patient was offered a second attempt at surgical resection 9 months after her initial surgical exploration. Her tumor was successfully resected without SMV resection or reconstruction and final pathology revealed a $2.0 \times 2.5 \times 2.2 \mathrm{~cm}$ mass confined to the pancreas with negative margins and 16 lymph nodes resected all negative for metastatic spread. She did well postoperatively with resolution of hyperglycemia allowing discontinuation of insulin and only occasional diarrhea due to pancreatic insufficiency which improved with pancreatic enzyme replacement. Three months after surgery, she remains free of disease recurrence by imaging and has had normalization of serum chromogranin A level to 206 $\mathrm{ng} / \mathrm{ml}$.

\section{Discussion}

PNET is a relatively uncommon tumor that arises in the endocrine cells of the pancreas and is also referred to as pancreatic islet cell tumor. There is variability in presentation partly dependent on if it is a functional (hormone-secreting) or nonfunctional tumor. Functional tumors can produce symptoms related to excess production of hormones such as gastrin, insulin, and glucagon. The majority of functional tumors are benign but are less prevalent than nonfunctional tumors which are more likely to be malignant. Nonfunctioning tumors typically remain silent until they cause symptoms due to mass effect similar to pancreatic adenocarcinoma. The overall annual incidence of PNETs from 1973 to 2000 was reported to be 0.22 cases per 100,000 with a yearly trend of increased incidence [1]. A more recent study confirms this trend with an annual incidence of 0.32 per 100,000 cases reported from 2000 to 2004 [2]. Nevertheless, this remains a rare tumor which limits the opportunity to establish clinical trial-based evidence for standard therapy.

Optimal treatment for localized PNET is curative surgical resection [3]. Until recently, the only standard option for advanced disease was a somatostatin analogue. For tumors that are localized with no evidence of distant spread, but are too advanced to surgically resect, there is no evidence to guide optimal management and therefore 
we are limited to extrapolating results obtained in the advanced metastatic disease setting.

Historically, somatostatin analogues have been utilized in functional tumors to control symptoms. Evidence for benefit beyond this was provided by a prospective, randomized placebo-controlled trial by the PROMID study group which reported an antiproliferative effect of octreotide LAR on neuroendocrine midgut tumors [4]. Stable disease was achieved in $66.7 \%$ of patients in the octreotide LAR group versus stable disease in $37.2 \%$ of patients in the placebo group with a median time to tumor progression of 14.3 months in the octreotide LAR group and 6 months with the placebo group [4]. These results helped solidify octreotide as a reasonable option for disease stabilization. Definitive overall survival benefit was limited by crossover of patients in the placebo group to somatostatin analogue treatment at tumor progression. Notably, this particular therapy does not achieve significant tumor regression. In our patient who was free of any symptoms and for whom the goal was to achieve tumor shrinkage, octreotide lacked any clear potential for achieving a clinical benefit.

More recently, two new drugs, everolimus and sunitinib, have each been approved for treatment of advanced PNETs. Everolimus, a mammalian target of rapamycin (mTOR) inhibitor, was shown to increase progression-free survival in a randomized, placebo-controlled trial [5]. Patients with advanced disease and radiologic evidence of disease progression were found to have an 11.4-month median progression-free survival with everolimus as compared to 5.4 months in the placebo group [5]. Analysis of secondary outcomes showed $5 \%$ of patients who received everolimus achieved a partial response by RECIST criteria [5]. The median duration of treatment with everolimus was 8.79 months. Dose adjustments, either reductions or temporary interruptions, were necessary in 59\% of patients and treatment discontinued in $13 \%$ due to adverse events. The most common drug-related adverse events reported were stomatitis, rashes, diarrhea and fatigue [5]. Everolimus was approved by the Food and Drug Administration (FDA) based on the above survival benefit and although it does not achieve a high rate of tumor shrinkage, it was considered an option in this patient.

Sunitinib is a multi-tyrosine kinase inhibitor which has also shown to have activity in patients with PNET. A randomized, double-blinded, placebo-controlled phase 3 trial was conducted and demonstrated that sunitinib improved progression-free survival when compared to placebo: median 11.4 months versus 5.5 months, respectively [6]. In addition, there was a $9.3 \%$ tumor response rate with a median time to tumor response of 3.1 months in patients treated with sunitinib [6]. The median duration of treatment with sunitinib was only 4.6 months with $22 \%$ of patients remaining on the study for over 1 year [6]. The most common adverse events leading to discontinuation of the drug were fatigue, diarrhea and cardiac failure with dose-limiting toxicity from neutropenia and diarrhea. Similar to everolimus, the survival benefit seen in this trial warrants consideration for a patient like ours but again, the $<10 \%$ partial response rate tempered our enthusiasm given our goal of down-staging her tumor.

Although both everolimus and sunitib provide benefit of increased progression-free survival, neither has been shown to achieve significant tumor shrinkage. Numerous phase II and multi-institutional trials have shown modest benefit with chemotherapeutic agents such as streptozocin, fluorouracil, doxorubicin, gemcitabine, oxaliplatin, temozolomide, thalidomide and capecitabine [7, 8]. The specific 
combination of capecitabine and tomozolomide has been shown in preclinical studies to have in vitro efficacy [9] and clinical experience was reported in a retrospective study suggesting activity with this well-tolerated combination [8]. In a study done at the Moffitt Cancer Center, a cohort of 30 patients with pancreatic endocrine carcinomas were treated with oral capecitabine $750 \mathrm{mg} / \mathrm{m}^{2}$ twice daily for 14 days and oral temozolomide $200 \mathrm{mg} / \mathrm{m}^{2}$ daily for 5 days every 28 days with ondansetron given prior to each dose of temozolomide to decrease nausea [10]. The radiographic response rate in patients with metastatic disease was $70 \%$ with a median progression-free survival of 18 months and median duration of radiographic response of 20 months [10]. The median duration of treatment was 8 cycles, with a range from 3 to 23 cycles. The majority of side effects were grade 1-2 thrombocytopenia, hand-foot skin reaction and fatigue. Our patient, with locally advanced disease, was treated with capecitabine and temozolomide for 8 total cycles with the goal of converting her disease to one that would be amenable to a curative surgical resection. She tolerated therapy well with minimal side effects and 9 months after initial exploratory surgery she ultimately underwent complete resection of a T2N0 tumor with 16 negative lymph nodes and negative margins.

Considering the relative rarity of PNETs, there has undoubtedly been significant progress in the development of treatment options just in the past few years. Everolimus and sunitinib provide options for agents that improve survival primarily through stabilization of disease but lack significant potential to cause tumor shrinkage relevant to locally advanced disease. Chemotherapy with capecitabine and temozolomide appears to be a viable option with potential for a high rate of response that proved to be particularly valuable in our young patient and warrants consideration for clinical trial exploration as neoadjuvant therapy for locally advanced, unresectable PNETs. 

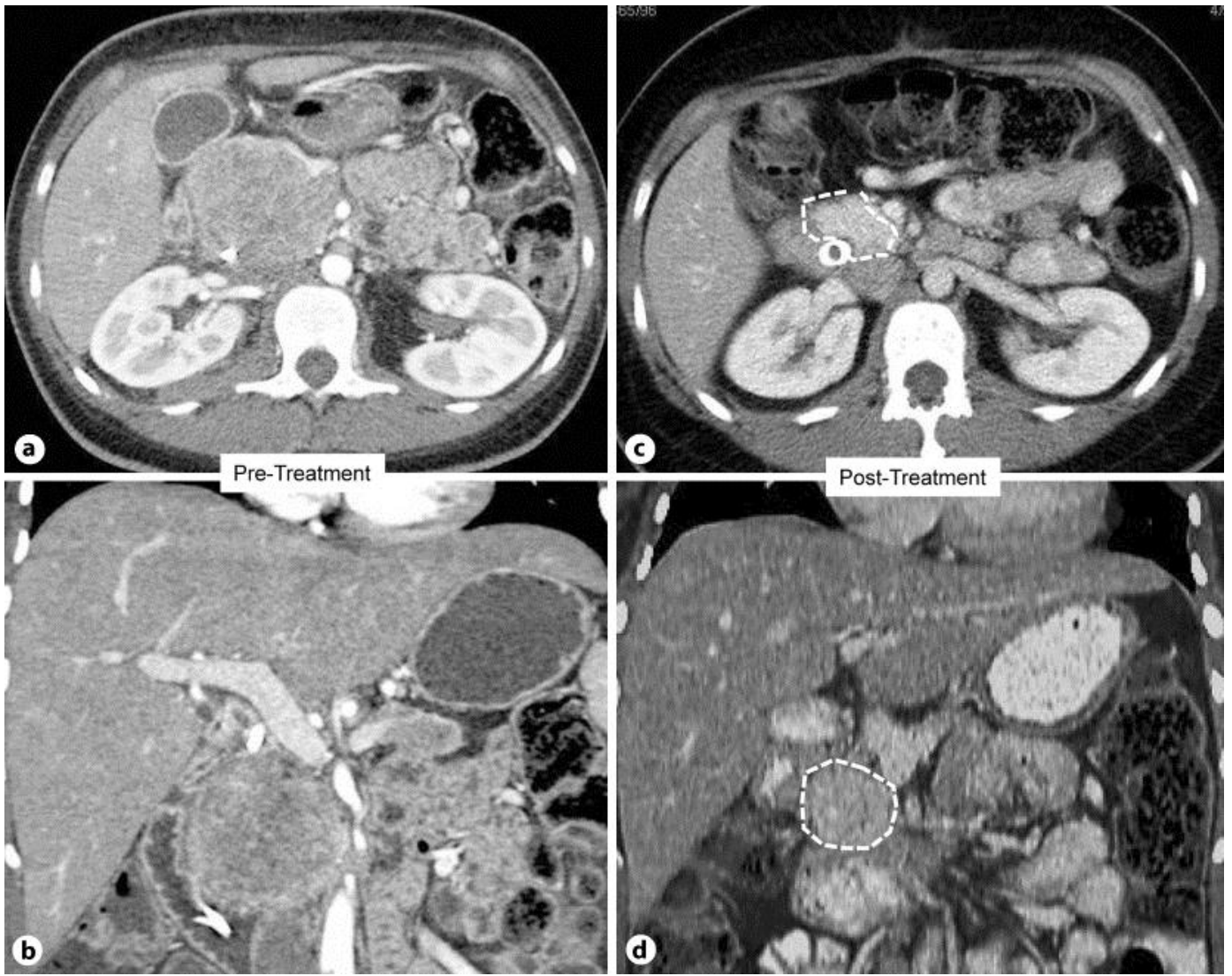

Fig. 1. CT findings of pancreatic mass. a, b Prior to neoadjuvant chemotherapy. c, d After 8 cycles of neoadjuvant everolimus and temozolomide.

\section{References}

1 Halfdanarson TR, Rabe KG, Rubin J, Petersen GM: Pancreatic neuroendocrine tumors (PNETs): incidence, prognosis and recent trend toward improved survival. Annals of Oncology 2008;19:1727-1733.

2 Yao JC, Hassan M, et al: One hundred years after 'carcinoid': epidemiology of and prognostic factors for neuroendocrine tumors in 35,825 cases in the United States. J Clin Oncol 2008;26:3063-3072.

3 Oberg K, Knigge U, et al: Neuroendocrine gastro-entero-pancreatic tumors: ESMO Clinical Practice Guidelines for diagnosis, treatment and follow-up. Ann Oncol 2012;23(suppl. 7):vii124-vii130.

4 Rinke A, Müller HH, et al: Placebo-controlled, double-blind, prospective, randomized study on the effect of octreotide LAR in the control of tumor growth in patients with metastatic neuroendocrine midgut tumors: a report from the PROMID Study Group. J Clin Oncol 2009;27:4656-4663.

5 Yao JC, Shah MH, Ito T, et al: Everolimus for advanced pancreatic neuroendocrine tumors. N Engl J Med 2011;364:514-523.

6 Raymond E, Dahan L, et al: Sunitinib malate for the treatment of pancreatic neuroendocrine tumors. N Engl J Med 2011;364:501-513.

7 Reidy-Lagunes DL: Systemic therapy for advanced pancreatic neuroendocrine tumors: an update. J Natl Compr Canc Netw 2012;10:777-783.

8 Isacoff WH, Moss RA, Pecora AL, Fine RL: Temozolomide/capecitabine therapy for metastatic neuroendocrine tumors of the pancreas. A retrospective review. J Clin Oncol 2006;24:14023.

9 Fine R, Fogelman D, Schreibman S: Effective treatment of neuroendocrine tumors with temozolomide and capecitabine. J Clin Oncol 2005;23:4216.

10 Strosberg JR, Fine RL, et al: First-line chemotherapy with capecitabine and temozolomide in patients with metastatic pancreatic endocrine carcinomas. Cancer 2011;117:268-275. 